\title{
The spectrum of resistance in SR/CR mice: the critical role of chemoattraction in the cancer/leukocyte interaction
}

\author{
Gregory Riedlinger1,2, Jonathan Adams¹, John R Stehle Jr1 , Michael J Blanks³, Anne M Sanders¹, Amy M Hicks', \\ Mark C Willingham ${ }^{1}$ and Zheng Cui*1,2
}

\begin{abstract}
Background: Spontaneous regression/complete resistance (SR/CR) mice are a unique colony of mice that possess an inheritable, natural cancer resistance mediated primarily by innate cellular immunity. This resistance is effective against sarcoma 180 (S180) at exceptionally high doses and these mice remain healthy.

Methods: In this study, we challenged SR/CR mice with additional lethal transplantable mouse cancer cell lines to determine their resistance spectrum. The ability of these transplantable cancer cell lines to induce leukocyte infiltration was quantified and the percentage of different populations of responding immune cells was determined using flow cytometry.

Results: In comparison to wild type (WT) mice, SR/CR mice showed significantly higher resistance to all cancer cell lines tested. However, SR/CR mice were more sensitive to MethA sarcoma (MethA), B16 melanoma (B16), LL/2 lung carcinoma (LL/2) and J774 lymphoma (J774) than to sarcoma 180 (S180) and EL-4 lymphoma (EL-4). Further mechanistic studies revealed that this lower resistance to MethA and LL/2 was due to the inability of these cancer cells to attract SR/CR leukocytes, leading to tumor cell escape from resistance mechanism. This escape mechanism was overcome by co-injection with S180, which could attract SR/CR leukocytes allowing the mice to resist higher doses of MethA and LL/2. S180-induced cell-free ascites fluid (CFAF) co-injection recapitulated the results obtained with live S180 cells, suggesting that this chemoattraction by cancer cells is mediated by diffusible molecules. We also tested for the first time whether SR/CR mice were able to resist additional cancer cell lines prior to S180 exposure. We found that SR/CR mice had an innate resistance against EL-4 and J774.

Conclusions: Our results suggest that the cancer resistance in SR/CR mice is based on at least two separate processes: leukocyte migration/infiltration to the site of cancer cells and recognition of common surface properties on cancer cells. The infiltration of SR/CR leukocytes was based on both the innate ability of leukocytes to respond to chemotactic signals produced by cancer cells and on whether cancer cells produced these chemotactic signals. We found that some cancer cells could escape from SR/CR resistance because they did not induce infiltration of SR/CR leukocytes. However, if infiltration of leukocytes was induced by co-injection with chemotactic factors, these same cancer cells could be effectively recognized and killed by SR/CR leukocytes.
\end{abstract}

\section{Background}

SR/CR mice are a unique colony of cancer-resistant mice derived from a single male BALB/c mouse that unexpectedly survived challenges with the extremely aggressive

* Correspondence: zhengcui@wfubmc.edu

1 Department of Pathology, Wake Forest University School of Medicine, Medical Center Blvd, Winston-Salem, North Carolina, 27157, USA Full list of author information is available at the end of the article mouse cancer cell line S180 at doses up to several million times greater than the lethal dose for WT mice [1-3]. This resistance was remarkable since S180-induced malignancy with rapid lethality has not been effectively treated previously by any other existing therapy. The SR/CR trait was found to be inheritable in an autosomal dominant manner and has subsequently been bred into over 2000 descendants in several inbred mouse strains. This domi- 
nant trait of cancer resistance is mediated primarily by leukocytes of the innate immune system and does not require any prior manipulation. However, the trait could not be mapped to a specific chromosome region after numerous attempts by either backcross or congenic breeding strategies (unpublished results), suggesting that the responsible genetic element may not reside at a fixed chromosomal location. When SR/CR mice are challenged repeatedly with S180, the composition of infiltrating leukocytes remains primarily leukocytes of innate immunity [4]. The leukocytes of SR/CR mice can be transferred to sensitive wild-type (WT) mice for prevention and treatment of established malignancies in immune-compatible recipients without adverse side-effects [4].

Another intriguing property of the resistance in SR/CR mice is that they are healthy throughout their lifespan suggesting that normal cells in these mice are not harmed by the anticancer response that targets cancer cells with exceptionally high specificity [2]. The cancer resistant phenotype can be retained for life if the mice are frequently challenged with cancer cells. Isolated SR/CR leukocytes display in vitro cytolytic activity against a wide array of lethal transplantable mouse cancer cell lines that are distinct in origin, morphology and cellular properties $[2,3]$. Meanwhile, transformed but non-cancerous cells, such as $\mathrm{CHO}$ or NIH-3T3, are not killed by the SR/CR leukocytes in vitro[4].

Upon challenge with S180 tumor cells, the response of SR/CR mice to cancer cells involves three sequential yet distinct cellular processes of the leukocytes [5]. First, there is a rapid infiltration of SR/CR leukocytes to the site of cancer. This process requires the leukocytes to sense a chemoattractant gradient before making unidirectional movement from their storage sites, such as the bone marrow, spleen, peripheral lymph nodes and circulation, toward the higher end of the chemoattractant gradient. Meanwhile, a clearly-defined chemoattractant gradient must be established by cancer cells with the higher end of the gradient at the cancer site. Second, upon arrival at the cancer site, SR/CR leukocytes make tight physical contact with the surface of cancer cells, exemplified by rosette formation between cancer cells and leukocytes. This process requires SR/CR leukocytes to recognize unique surface properties of live cancer cells to allow surface binding between the plasma membranes of leukocytes and cancer cells. Third, upon surface contact, leukocytes deliver effector mechanisms to cause damage to the plasma membranes, swelling and eventual rupture of cancer cells. This final process involves, but is not limited to, several known common effector mechanisms, such as degranulation of neutrophilic granulocytes. The third process is not unique to SR/CR mice since these effector mechanisms have been reported previously in WT mice in the killing of cancer cells [6-8]. However, the first two processes were unique to SR/CR mice since WT leukocytes are unable to respond to the same cancer cells with either infiltration or rosette formation.

Based on the in vitro observations and other in vivo observations, this unique phenotype appears to be a true general resistance against cancer cells that may extend to additional mouse cancer cell lines. It would be helpful in elucidating the mechanism of SR/CR resistance if we were to identify cancer cell lines that could escape this resistance. Here, we report our findings.

\section{Methods}

\section{Cell Lines and Mouse Strains}

S180, EL-4, LL/2, B16, and J774 cell lines were purchased from American Type Culture Collection and propagated in culture according to the manufacturer's protocol. MethA were a kind gift from Dr. Lloyd Old (Ludwig Institute for Cancer Research, New York). BALB/c mice were purchased from Charles River Laboratory and C57BL/6 mice were purchased from the Jackson Laboratory. SR/ CR mice $(1,4)$ were bred at the Animal Research Programs of Wake Forest University (WFU) Health Sciences. Mice were housed in plastic cages covered with individual air filter tops, containing corncob shavings as bedding, allowed free access to water and regular chow and exposed to a 12-hr fluorescent light/dark cycle. All animal procedures were conducted according to Institutional Animal Care and Use Committee guidelines and the National Institutes of Health Guide for the Care and Use of Animals and with all protocols and procedures approved by the IACUC of the WFU Health Sciences. Mature mice ( 2-6 months old) were used for all experiments unless specifically noted elsewhere.

\section{SR/CR In Vivo Resistance}

$\mathrm{SR} / \mathrm{CR}$ and WT mice were injected with the indicated number of cancer cells i.p. and the ability of mice to resist the cells was determined by survival. Moribund mice were euthanized. Cell-free ascites fluid (CFAF) was obtained by collection of ascites fluid from WT mice that developed S180 induced ascites. This ascites fluid was spun twice at $400 \mathrm{~g}$ for 5 minutes each, followed by one spin at $3000 \mathrm{~g}$ for 5 minutes with the cell free supernatant collected after each spin. This CFAF was then used fresh for subsequent experiments. In the co-injection experiments, $2 \mathrm{ml}$ of CFAF was co-injected with MethA or LL/2 and these mice then received weekly injections of CFAF for the duration of the experiment.

\section{Immune Infiltration in Response to Cancer Cell Lines and CFAF}

SR/CR or WT mice were injected i.p. with the indicated cancer cell line or CFAF. Heat inactivated CFAF was obtained by heating CFAF for 15 minutes at $100^{\circ} \mathrm{C}$ in a 
water bath. Fractions of CFAF containing molecules $>$ or $<5 \mathrm{kD}$ were obtained using Amicon Ultra $5 \mathrm{k}$ centrifugal devices (Millipore, Billerica, MA) according to the manufacturer's instructions. Briefly, total CFAF was placed in the upper chamber of the centrifugal device and then spun at $3000 \mathrm{~g}$ for 20 minutes separating the $>5 \mathrm{kD}$ fraction into the top chamber and the $<5 \mathrm{kD}$ fraction into the bottom of the tube. A heat inactivated sample of the $<5$ $\mathrm{kD}$ fraction was obtained by heating this fraction for 15 minutes at $100^{\circ} \mathrm{C}$ in a water bath. Six hours after injection the peritoneal cavity was lavaged and the number of cells was quantitated using a cytometer. For each injection group, $\mathrm{n}>=4$.

\section{Flow Cytometry}

Cells from peritoneal lavages were stained with specific antibodies to the cell surface markers Ly6G, NK1.1, F4/ 80, CD11c, CD19, CD4, and CD8 (BD Pharmingen, San Diego, CA) according to standard procedures recommended by Pharmingen. Briefly, 400,000 cells from the peritoneal lavage were added in $100 \mu \mathrm{l}$ of FMF medium (PBS w/1\% FBS) in $12 \times 75 \mathrm{~mm}$ flow tubes that were spun at $400 \mathrm{~g} \times 5$ minutes. The supernatant was removed and the cells were resuspended in $50 \mu \mathrm{l}$ of $5 \mu \mathrm{g} / \mathrm{ml} \mathrm{FITC-con-}$ jugated antibody (Ly6G, NK1.1, F4/80, CD11c, CD19, CD4, or CD8) or FMF medium alone as a control and incubated 30 minutes on ice in the dark. The cells were then washed twice with $100 \mu \mathrm{l}$ of FMF medium and then resuspended in $300 \mu \mathrm{l}$ of FMF medium keeping the samples in the dark as much as possible. The samples were then analyzed on a FACSCalibur flow cytometer (BD Bioscience, Mountain View, CA). Forward and side scatter gain settings were tuned to sort live cells from cell fragments. The total value for these seven markers was arbitrarily set at $100 \%$ for each sample to allow comparison between groups and for each injection group $n>=4$. S180, MethA, and LL/2 were each tested and found to have no reactivity to any of the cell surface markers used.

\section{Results \\ SR/CR Mice Resist a Broad Array of Lethal Mouse Cancer Cell Lines}

To determine the respective maximum tolerated doses (MTD), we challenged SR/CR mice and control WT mice with various lethal mouse cancer cell lines at different doses and measured the disease-free survival of the mice. SR/CR mice were first identified by their ability to survive 2 standard screens with $2 \times 10 \mathrm{e} 5$ and $5 \times 10 \mathrm{e} 6$ S180 i.p., which always induced lethality in WT mice. The mice were then divided into groups that were challenged with other cancer cells at different doses. SR/CR mice in the $\mathrm{BALB} / \mathrm{c}$ background were challenged with J774 lymphoma (MHC haplotype H2d) or MethA sarcoma (H2d) cells. SR/CR mice in the C57BL/6 congenic background (generations $\mathrm{n} 8$ or later) were challenged with EL-4 lymphoma (H2b), LL/2 lung carcinoma (H2b), B16 melanoma (H2b) or S180 sarcoma (H2q). All WT mice died with i.p. doses of $10 \mathrm{e} 3$ cells or fewer with all cancer cell lines tested. The MTD of LL/2 for WT mice was less than 100 cells. As few as one viable EL-4 has been reported previously to be lethal in WT mice [9]. In the BALB/c background, all SR/CR mice survived $5 \times 10 \mathrm{e} 4 \mathrm{J774}$ before they began to die at higher doses, while $70 \%$ of mice challenged with $10 \mathrm{e} 5$ MethA survived. In the C57BL/6 background, all SR/CR mice survived with $8 \times$ $10 \mathrm{e} 7 \mathrm{~S} 180$ before they began to die at higher doses. No death in SR/CR mice was observed with doses up to $2 \times$ $10 \mathrm{e} 8$ for EL-4. The survival of C57BL/6 SR/CR mice challenged with B16 and LL/2 was greatly reduced with $40 \%$ surviving when challenged with $10 \mathrm{e} 3$ cells in both groups (Figure 1). Although no mice survived challenge with B16 or LL/2 at higher doses, the average survival time of SR/ $\mathrm{CR}$ mice at $5 \times 10 \mathrm{e} 4 \mathrm{~B} 16$ was greatly increased ( 34 days) compared to WT mice ( 21 days).

\section{Resistance of naïve SR/CR pups to cancer cells other than S180}

We injected naïve pups, age 6-8 weeks, with cancer cell lines other than S180 initially to determine if resistance to these cell lines was simply a result of cross-vaccination from antigens shared with S180 or if it was a result of innate recognition of a common surface property shared between different cancer cell lines. SR/CR mice had never previously been tested for their ability to resist additional cancer cell lines without first being able to survive challenge with S180. The naïve pups were obtained from our routine breeding scheme in which one C57BL/6 SR/CR parent is crossed with a $\mathrm{C} 57 \mathrm{BL} / 6 \mathrm{WT}$ parent. In this breeding scheme, $30-40 \%$ of the naive pups were expected to survive the initial challenge with S180. Five weeks after the naïve pups were given $10 \mathrm{e} 6 \mathrm{EL}-4$ as their first cancer challenge, 22 of 35 pups (65\%) survived and remained apparently healthy (Figure 2 ). When naïve pups were first screened with J774 at $5 \times 10 \mathrm{e} 4,1$ of 9 pups survived.

\section{S180 or CFAF Enhances the Resistance of SR/CR mice to MethA and LL/2}

It was somewhat surprising that the resistance of SR/CR mice against some other cancer cell lines was so much weaker than that against S180 and EL-4, whereas our in vitro assay results show that the SR/CR leukocytes could kill all of these cancer cell lines with similar efficiencies [[2], Z.C. unpublished results]. The increased sensitivity of SR/CR mice to MethA and LL/2 suggested that a failure might have occurred at one or more of the three required stages of leukocyte action for the anticancer response. We set out to examine which of the three 


\section{Cell Line Survival}

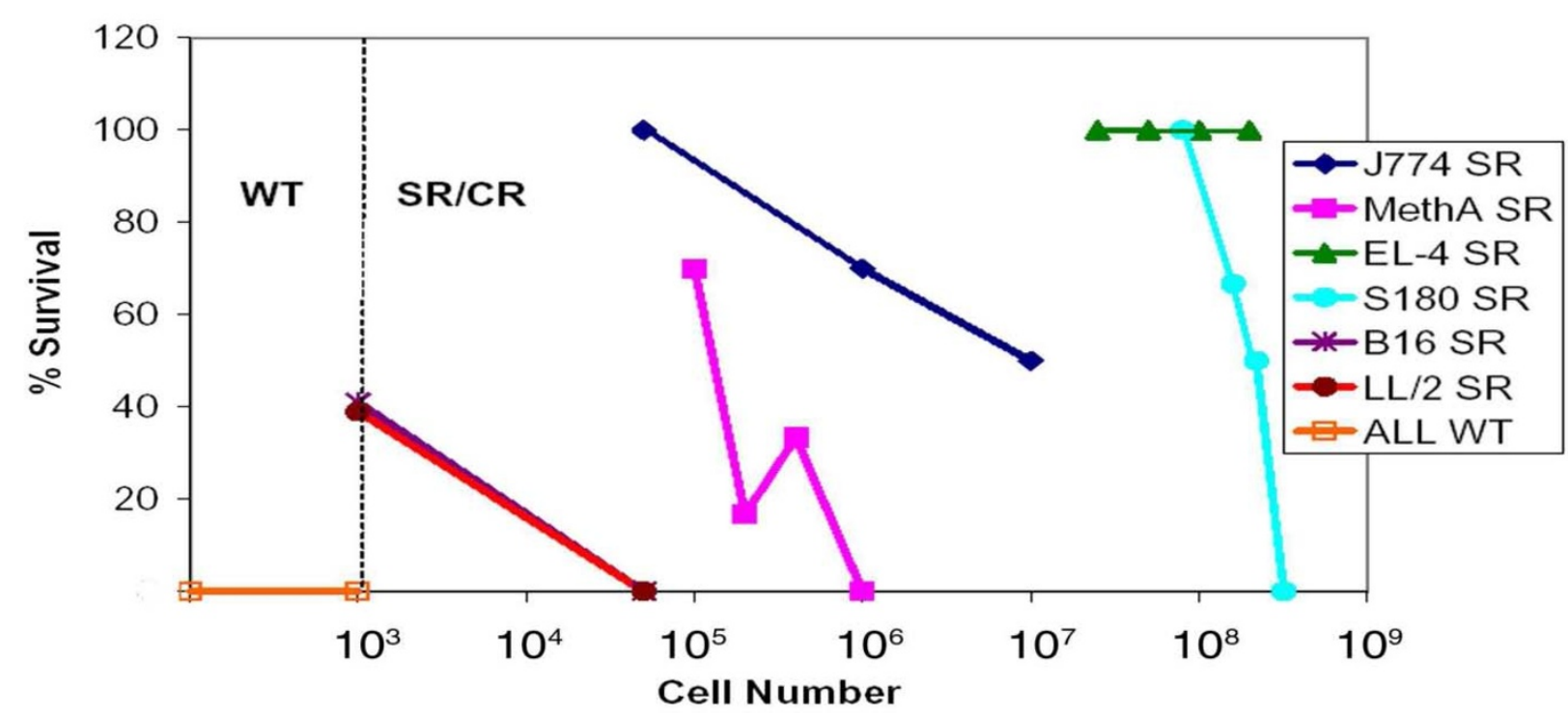

Figure $1 \mathrm{SR} / \mathrm{CR}$ mice resist a broad range of lethal cancer cell lines at different doses. The percentage of SR/CR mice that were able to survive challenge with J774, MethA, EL-4, S180, B16, or LL/2 was determined empirically. Mice were monitored for at least 60 days after challenge. SR/CR C57BL/6 mice were challenged with $10 \mathrm{e} 3$ or $5 \times 10 \mathrm{e} 4 \mathrm{~B} 16$ or LL/2. SR/CR C57BL/6 mice were also challenged with $0.8 \times 10 \mathrm{e} 7,1.6 \times 10 \mathrm{e} 8,2.2 \times 10 \mathrm{e} 8$, or $3.2 \times 10 \mathrm{e} 8 \mathrm{~S} 180$ or $2.5 \times 10 \mathrm{e} 7,5 \times 10 \mathrm{e} 7,10 \mathrm{e} 8$, or $2 \times 10 \mathrm{e} 8 \mathrm{EL}-4$. SR/CR BALB/C mice were challenged with 10e5, $2 \times 10 \mathrm{e} 5,4 \times 10 \mathrm{e} 5$, or 10e $6 \mathrm{MethA}$ or $5 \times 10 \mathrm{e} 4,10 \mathrm{e} 6$, or $10 \mathrm{e} 7 \mathrm{~J} 774$. WT mice uniformly died at all doses with all cell lines tested. At least 5 mice were tested for any given dose of tumor cells injected.

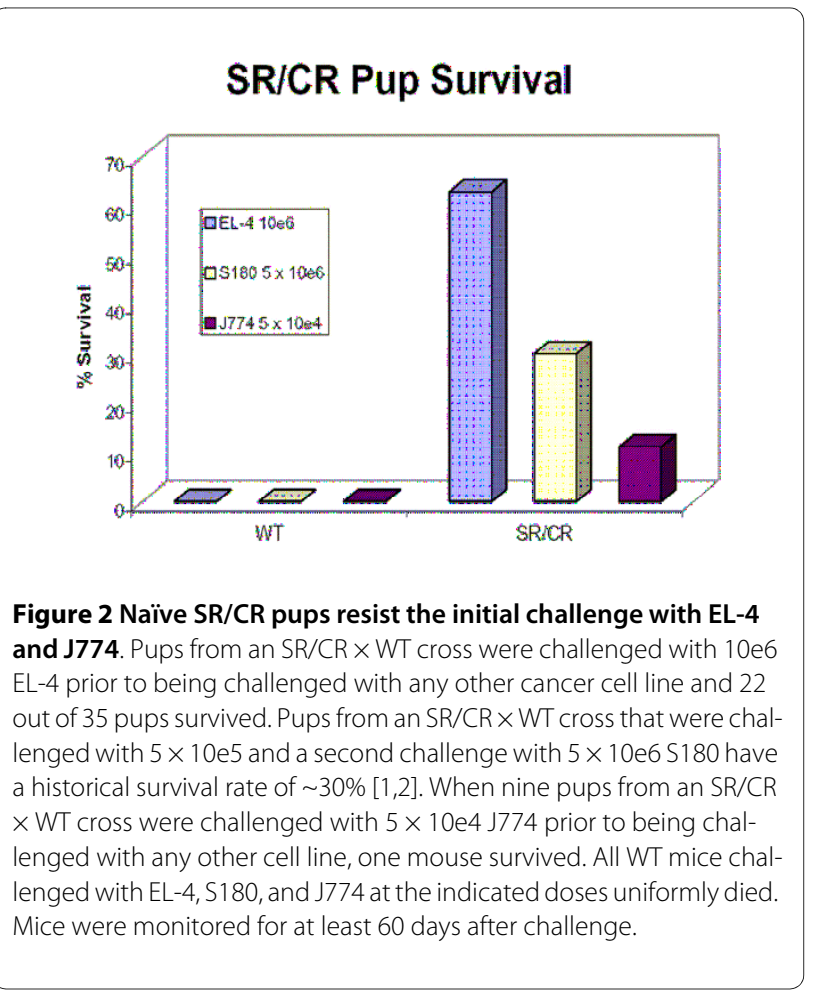

required stages of leukocyte action was responsible for this failure of the anticancer response. If the increased sensitivity was caused by a failure of LL/ 2 cells in immune attraction, the resistance should be restored in SR/CR mice when LL/2 were co-injected with live cancer cells that were known to induce leukocyte infiltration, such as S180. To test this hypothesis, we mixed $2 \times 10 \mathrm{e} 5 \mathrm{LL} / 2$ cells with $10 \mathrm{e} 6 \mathrm{~S} 180$ cells and co-injected them i.p. into SR/CR mice. All of the co-injected SR/CR mice survived, whereas all of the co-injected WT mice died. A nearly identical result was achieved when LL/2 was co-injected with $10 \mathrm{e} 6$ irradiated S180 (25 Gy) that were alive for several days but were not proliferating. To further test the hypothesis that the resistance to LL/ 2 at $2 \times 10 \mathrm{e} 5$ was restored by simply provoking leukocyte migration, we examined if S180-induced CFAF could achieve the same effect as the live or irradiated S180. CFAF (cell-free ascites fluid) is the immediate liquid environment produced as the S180 cells grow in the peritoneum of WT mice. If the enhanced survival from the co-injection was truly mediated by leukocyte migration as a result of diffusible chemoattractants from S180, CFAF should contain all the diffusible chemoattractants and should be able to recapitulate the enhanced survival observed with live S180 cells to some extent. Indeed, when $2 \times 10 \mathrm{e} 5 \mathrm{LL} / 2$ were co-injected with CFAF (with $2 \mathrm{ml}$ weekly), a clear 


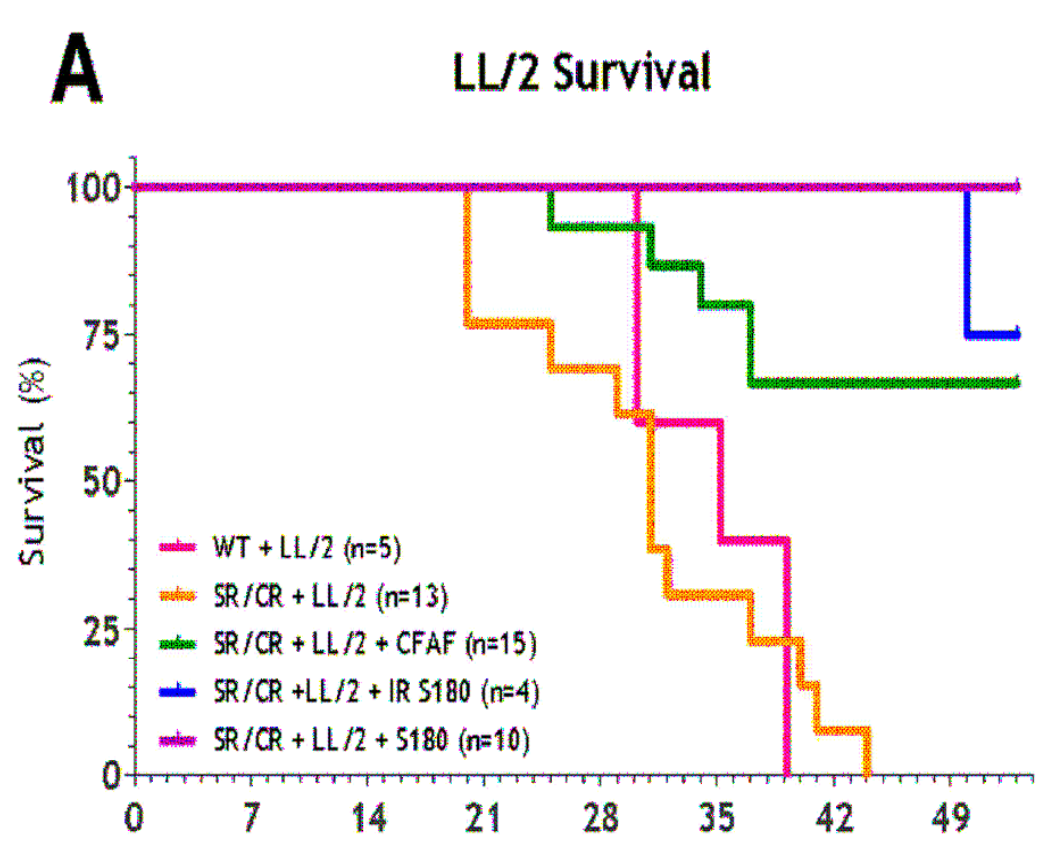

Days Post-Tumor Injection
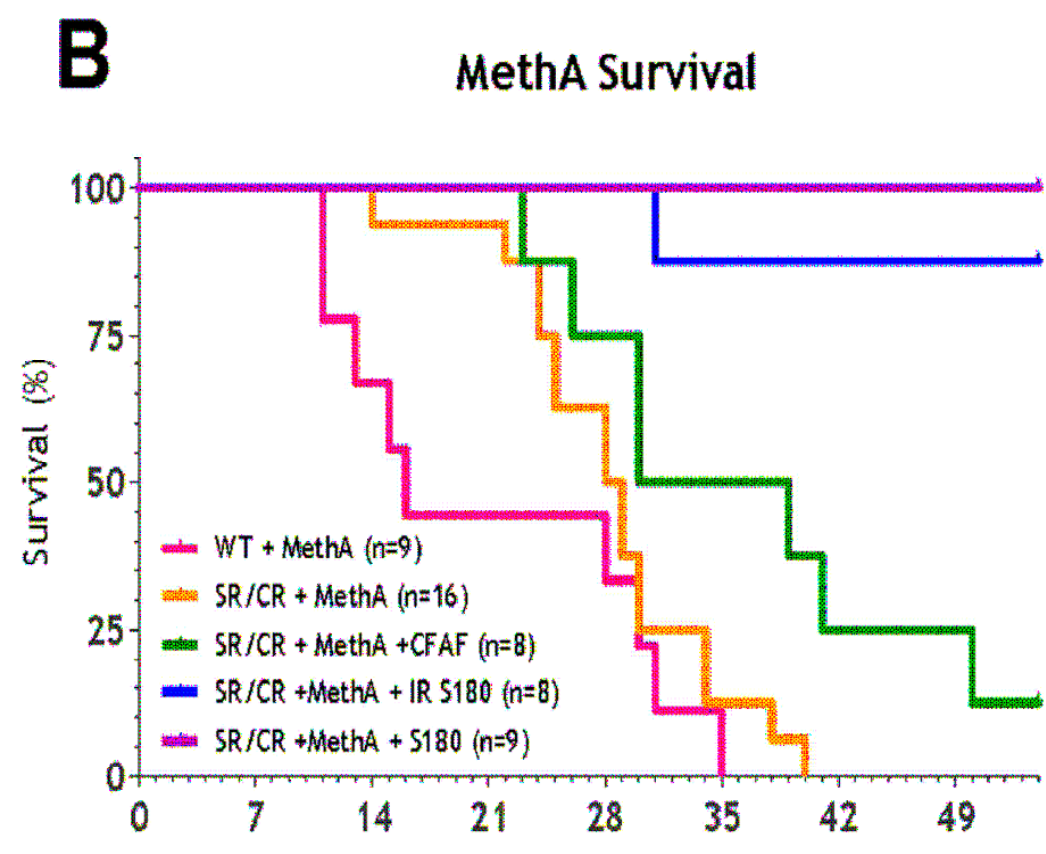

Days Post-Tumor Injection

Figure 3 S180 or cell free ascites fluid (CFAF) enhances SR/CR resistance. SR/CR or WT mice were challenged with $2 \times 10$ e5 LL/2 (upper panel) or 10e6 MethA (lower panel) i.p. either alone or with 10e6 S180, 10 e6 irradiated S180, or weekly injections of CFAF and survival curves were generated. The number of mice in each group is indicated in the figure. 
survival benefit was observed (Figure 3 upper panel). A similar result was obtained for the resistance to MethA co-injected with S180, irradiated S180 and CFAF (Figure 3 lower panel) and preliminary results indicate that S180 also enhances the survival of SR/CR mice challenged with higher doses of B16 (data not shown).

\section{S180 only enhances the resistance of SR/CR mice to $L L / 2$ locally}

The improved survival in SR/CR mice co-injected with S180 or CFAF could be the consequence of two distinct events; higher infiltration of SR/CR leukocytes or improved effector function by systemic activation of SR/ CR leukocytes induced by S180 coinjection. To determine which of the processes predominated, we examined the ability of S180 to enhance SR/CR resistance to LL/2 when injected in the same site or at a remote site (Figure 4). Our results indicate that $\mathrm{S} 180$ only enhances resistance to $\mathrm{LL} / 2$ when injected at the same site.

\section{S180 and CFAF induce leukocyte infiltration in SR/CR mice}

The need for co-injection suggested that the ability to induce leukocyte infiltration along with the number of infiltrating leukocytes may be directly related to the number of cancer cells the SR/CR mice can resist, with a strong infiltration resulting in a higher MTD. We hypothesized that the low-MTD cancer cells (LL/2 and MethA) should have lower activity for inducing SR/CR leukocyte infiltration, whereas the high-MTD cancer cells (S180) should have higher activity for inducing leukocyte infil-

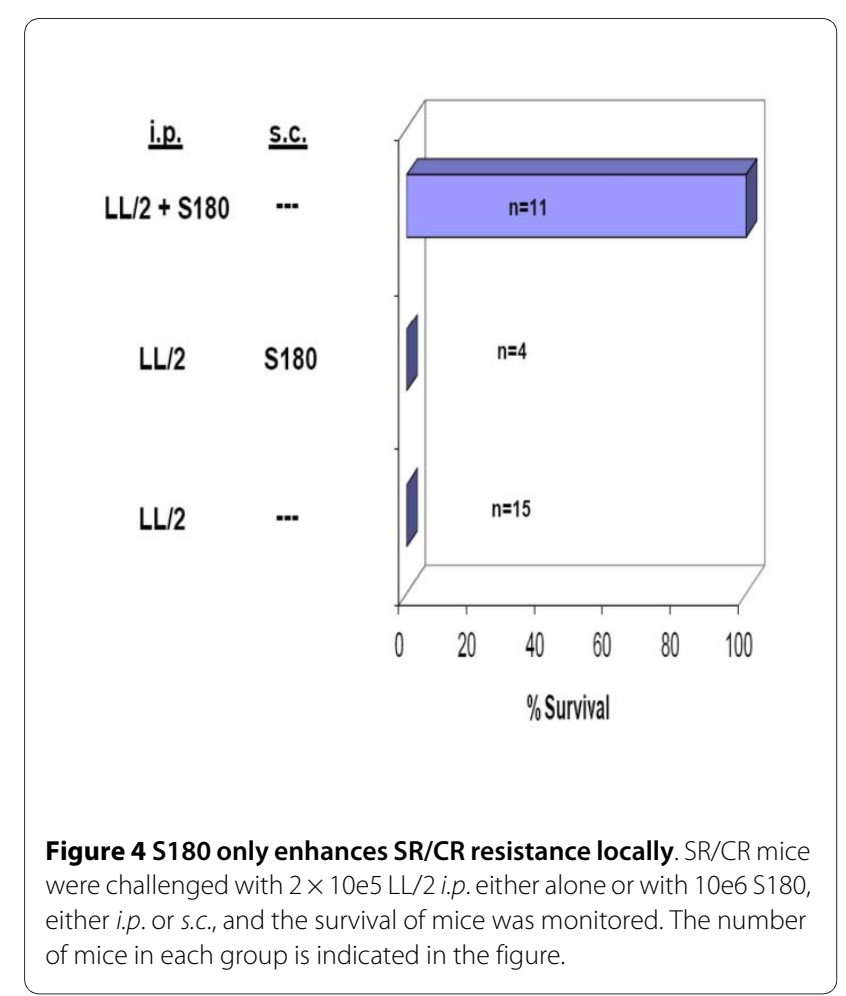

tration. To test this hypothesis, we quantified the number of infiltrating cells in the peritoneum and used flow cytometry to profile the cell types of the infiltrating leukocytes based on surface markers six hours after being challenged with different cancer cells. Aliquots of leukocytes were specifically labeled with surface antibodies for neutrophils (Ly6G positive), macrophages (F4/80 positive), natural killer cells (NK1.1 positive), dendritic cells (CD11c positive), B cells (CD19 positive), helper $\mathrm{T}$ cells (CD4 positive), and cytolytic T cells (CD8 positive) cells and then subjected to analysis by flow cytometry. The sum of these seven markers was arbitrarily set at $100 \%$ to allow comparison between different groups. The most noticeable differences were in the numbers of neutrophils and macrophages in response to S180 or CFAF between WT and SR/CR mice, while levels of $B$ cells remained fairly consistent (Figure 5A). It was clear that S180 induced significantly more leukocyte infiltration in SR/ CR mice than MethA and LL/2, and the percentage of leukocytes was shifted towards innate immune cells, specifically neutrophils and macrophages. It was interesting to note that this effect was specific for SR/CR mice since cancer cells or CFAF appeared to have an inhibitory effect on WT leukocyte infiltration (Figure 5C). CFAF provoked the largest leukocyte infiltration, consistent with the idea that the highest concentration of diffusible chemoattractants would accumulate in the peritoneal fluid over several days in the presence of a large number of live S180 cells. We also characterized some of the biochemical properties of the chemoattractants in S180induced CFAF. When CFAF was fractioned through an Amicon Ultra $5 \mathrm{k}$ centrifugal device, most of the chemoattraction activity was recovered in the filteredthrough fraction, suggesting that the chemoattractants were smaller than 5 kilo-Dalton. Furthermore, boiling CFAF for 10 min abolished most of the chemoattracting activity, suggesting that the chemoattractants were heat sensitive (Figure 5D).

\section{Discussion}

In the present study, we characterized the ability of SR/ $\mathrm{CR}$ mice to resist additional lethal cancer cell lines in vivo, and showed that SR/CR mice are able to resist these cell lines at higher doses than WT mice. However, SR/CR mice were able to resist some cancer cell lines at exceptionally high doses (high-MTD), whereas other cancer cell lines were only resisted at moderate or lower doses (low-MTD). We found that co-injection with either S180 or CFAF, both capable of inducing massive leukocyte infiltration specifically in SR/CR mice, was able to increase the level of resistance of SR/CR mice to cell lines with otherwise low-MTD.

Cancer cells may undergo selection to avoid detection by the immune system, a process termed cancer 

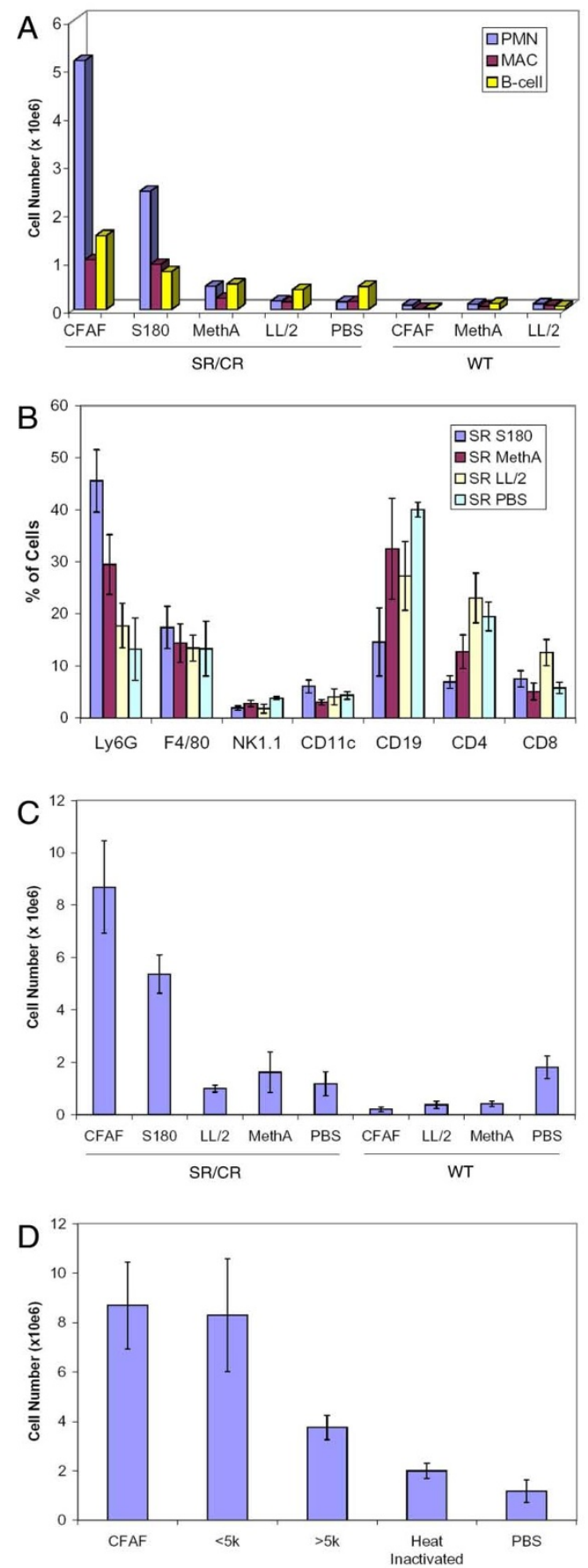

Figure 5 Leukocyte subset composition during challenge with different cancer cell lines. A) SR/CR and WT mice were challenged with $10 \mathrm{e} 5$ cells of the indicated cancer cell line, CFAF, or PBS and responding leukocytes were washed out at six hours, quantitated, stained with Ly6 $\mathrm{g}$ (PMN), F4/80 (Mac), and CD19 (B-cell) and profiled by flow cytometry. B) Composition of infiltrating immune cells. SR/CR mice were injected with 10e5 cells of the indicated cancer cell line or PBS and six hours later the peritoneal cavity was washed out. The peritoneal infiltrate was stained with Ly6 $\mathrm{g}$ (PMN), F4/80 (Mac), NK1.1 (NK cell), CD11c (dendritic cell), CD19 (B-cell), CD4, and CD8 and profiled by flow cytometry. The total value for these seven markers was arbitrarily set at $100 \%$ for each sample to allow comparison between groups. Values represent the mean $+/-$ SEM. For each injection group, $\mathrm{n}>=$ 4. C) Immune infiltration in response to cancer cell lines and CFAF. SR/CR or WT mice were injected i.p. with 10 e 5 cells of the indicated cancer cell line or CFAF and six hours later the peritoneal cavity was washed out and the number of cells was quantitated using a cytometer. Values represent the mean $+/$ - SEM. For each injection group, $n>=4$. D) SR/CR immune infiltration in response to CFAF, heat inactivated CFAF, and small ( $<5 \mathrm{kD})$ or large $(>5 \mathrm{kD}$ ) molecules found in CFAF fluid. SR/CR mice were injected as indicated and six hours later the peritoneal cavity was washed out and the number of cells was quantitated using a cytometer. Values represent the mean $+/$ - SEM. For each injection group, $n>=4$. 
immuno-editing that may drive the progression of malignancy [10]. A variety of tumor-derived factors may contribute to immunosuppressive processes that may extend immune evasion from the primary site to peripheral sites in patients with cancer [11]. The results from our experiments suggest that cancers with low-MTD escape from SR/CR anticancer immunity because they do not produce sufficient chemoattractants. These low-MTD cancer cell lines are capable of being eradicated in SR/CR mice at lower doses, presumably by the less than $2 \times 10 \mathrm{e} 6$ resident leukocytes usually present locally in the peritoneal cavity. At higher doses, however, the growth of these lowMTD cancer cell lines may outpace the killing ability of these limited resident leukocytes. This is in contrast to cell lines such as S180 that are able to induce a very large infiltration of leukocytes. The larger numbers of infiltrating SR/CR leukocytes are apparently capable of killing a much larger number of cancer cells.

Although the outcome of the co-injection experiment with S180 and CFAF was somewhat unexpected, the results, coupled with our previous work demonstrating that S180, MethA, and LL/2 were killed in an in vitro assay [2], suggest that the ability to induce leukocyte infiltration may be the most significant factor between these cancer cells in their ability to be resisted by SR/CR mice. There appear to be common surface properties that allow a variety of cancer cells to be recognized, bound, and destroyed by SR/CR leukocytes when they are in close proximity (Figure 6). Our results demonstrate that S180 only enhances resistance against LL/2 locally in SR/CR mice and argues that the ability of S180 to induce leukocyte infiltration is the critical event in augmenting resistance. While immune infiltration is clearly important for the eradication of cell lines such as LL/2 and MethA, we cannot completely exclude the possibility that co-injection with S180 or CFAF also activates the effector mechanism of SR/CR leukocytes, in addition to the induction of their infiltration.

The results also suggest that there is a diffusible chemoattractant gradient established by some cancer cells. CFAF from S180 contains high levels of these chemoattractants that are specific for SR/CR leukocytes. Our results indicate that the most active chemoattractants are molecules smaller than $5 \mathrm{kD}$ that are heat sensitive. It is currently unclear if these diffusible chemoattractants were produced by active cellular secretion $[11,12]$, by passive "surface shedding", a physical process, from cancer cells [13-16] or indirectly by the interaction between cancer cells and stromal tissues. Nevertheless, CFAF offers a good platform for further biochemical purification and identification of these chemoattractants. Apparently, chemoattraction of SR/CR leukocytes is a separate process from recognition of the common cancer cell surface properties that allow for

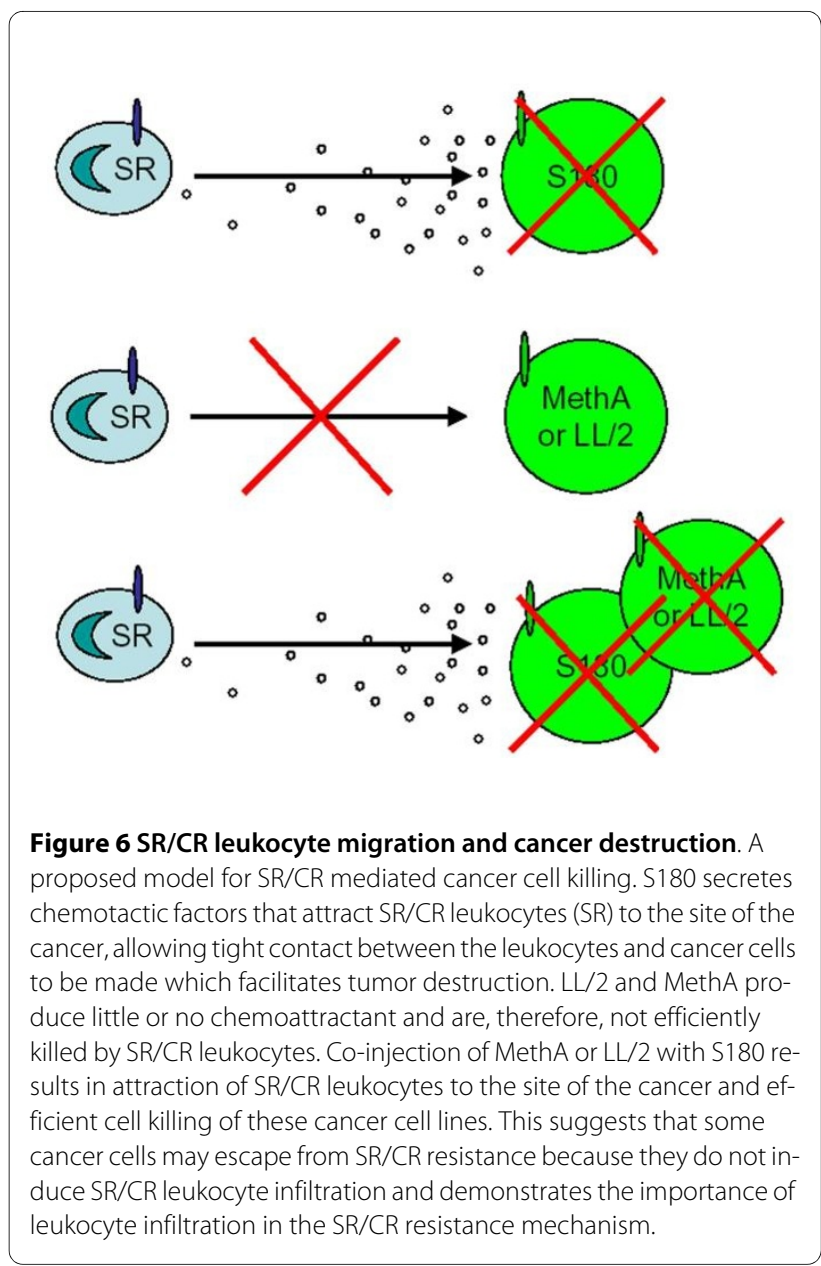

local binding and eradication of SR/CR leukocytes, since some cancer cells can lose the former process while retaining the properties of the latter. This ability to induce leukocyte infiltration through chemoattraction appears to be the reason that SR/CR leukocytes are effective as a systemic therapy against established S180 cancers, but are only locally effective against cancers like LL/ 2 .

The cancer/immune cell interaction involves events on either side that influence the ability of the immune system to eradicate the cancer. On the cancer side, malignant lesions may range from being highly immuneattractive (S180) to inducing little immune infiltration (LL/2). Cancer cells may develop mechanisms that prevent migration of leukocytes to the site of the cancer, either by turning off the production of inflammatory molecules that can act as chemoattractants or by producing molecules that actively inhibit immune cells, such as TGF- $\beta$ [17]. Our results indicate that cancer cells and CFAF may also produce molecules that are directly inhibitory to WT leukocytes (Figure 4C). On the host side, leukocytes themselves can vary significantly in any of the three required stages of leukocyte response. If leukocytes 
have a defect in infiltration to the cancer site, recognition of the cancer, or deployment of their effector mechanisms an effective anticancer response to protect the host cannot take place. For example, leukocytes may infiltrate the cancer site but may be unable to recognize and kill the cancer cells. This has been reported in some melanoma patients, in whom despite having melanoma-specific $\mathrm{T}$ cells infiltrating the tumor lesions, tumor rejection rarely occurs [18]. Additionally, the responsiveness of host leukocytes may be influenced by genetics, aging and environmental factors.

Our SR/CR model system is very interesting in light of many recent reports that there is a positive correlation between tumor infiltrating lymphocytes and the survival of patients with melanoma, ovarian cancer, bladder cancer, glioma, and colon cancer [19-23]. It is worth noting that in these examples of human cancers the correlation is with cells of the adaptive immune system, specifically $\mathrm{T}$ lymphocytes, while in the SR/CR mouse the resistance mechanism is mediated by the innate immune system. A recent study performed by Galon et al. is particularly intriguing as it specifically links activation of the cellular immune response, including macrophages of the innate immune system, to patient outcomes in colorectal cancer [24]. They find a significant correlation between expression of genes of the Th1 response and a beneficial outcome in risk of relapse after complete removal of the tumor.

The best case scenario for an anticancer protection mechanism in a host would be having cancer cells that secrete a chemoattractant and having leukocytes that can infiltrate, recognize the cancer cells as foreign, and completely destroy the cancer. However, the absence of one or more of these factors could lead to an unfavorable host/ cancer interaction enabling the cancer to escape immunosurveillance resulting in progression of the disease. When there is no recognition of cancer cells by leukocytes, manipulation of other processes, such as infiltration, will not improve host survival. However, if infiltration is the only deficiency, as we observe in SR/CR mice challenged with LL/2, local delivery of host leukocytes or establishment of a chemoattractant gradient at the cancer site could achieve therapeutic benefit.

\section{Conclusions}

Our results show that SR/CR mice had significantly higher resistance to all cancer cell lines tested in comparison to wild type (WT) mice. However, there was great variation in the number of cells that could be resisted by SR/CR mice across the different cancer cell lines tested. It appears that this variation is based on the ability of the cancer cell lines to induce leukocyte infiltration, as coinjection with chemoattractant factors increased the number of cancer cells that the SR/CR mice could resist.
We also demonstrate for the first time that SR/CR mice are able to resist cell lines other than S180 on the initial challenge. Therefore, it appears that the resistance against other cancer cells is also innate in nature since it does not require specific priming with non-self antigens found on S180. This supports the idea that SR/CR mice innately recognize a factor that is common on multiple cancer cell lines but absent on non-neoplastic cells.

\section{Competing interests}

The authors declare that they have no competing interests.

\section{Authors' contributions}

GR helped conceive of the study, participated in its design and coordination, helped perform the injections, lavages, flow cytometry, and drafted the manuscript. JA helped perform the injections, lavages, animal care and helped with manuscript preparation. JS helped design the studies, perform the lavages, irradiation, flow cytometry, and with manuscript and figure preparation. MB helped design the studies, perform the lavages/injections, and with manuscript and figure preparation. AS helped design the studies, perform the lavages/injections, and with manuscript preparation. $\mathrm{AH}$ helped design the studies, perform the lavages/injections, and with manuscript preparation. MW helped design the studies, helped with the histological evaluation, and with manuscript preparation. $Z C$ was responsible for the oversight of the entire project and including experimental design and manuscript writing. All authors read and approved the final manuscript.

\section{Acknowledgements}

The described studies were supported by grants from the Cancer Research Institute, the National Cancer Institute, and the Charlotte Geyer Foundation (to Z.C.). G.R. is supported by National Cancer Institute Training Grant CA79448.

\section{Author Details}

1Department of Pathology, Wake Forest University School of Medicine, Medical Center Blvd, Winston-Salem, North Carolina, 27157, USA, 2Department of Cancer Biology, Wake Forest University School of Medicine, Medical Center Blvd, Winston-Salem, North Carolina, 27157, USA and ${ }^{3}$ Molecular Genetics and Genomics Program, Wake Forest University School of Medicine, Medical Center Blvd, Winston-Salem, North Carolina, 27157, USA

Received: 28 September 2009 Accepted: 3 May 2010 Published: 3 May 2010

\section{References}

1. Koch J, Boschian A, Hau J, Rieneck K: Frequency of the cancer-resistant phenotype in SR/CR mice and the effect of litter seriation. In Vivo 2008, 22:565-569.

2. Cui Z, Willingham MC, Hicks AM, Alexander-Miller MA, Howard TD, Hawkins GA, Miller MS, Weir HM, Du W, DeLong CJ: Spontaneous regression of advanced cancer: identification of a unique genetically determined, age-dependent trait in mice. Proc Natl Acad Sci USA 2003, 100:6682-6687.

3. Cui Z: The winding road to the discovery of the SR/CR mice. Cancer Immun 2003, 3:14.

4. Hicks AM, Riedlinger G, Willingham MC, Alexander-Miller MA, Von KapHerr C, Pettenati MJ, Sanders AM, Weir HM, Du W, Kim J, et al:: Transferable anticancer innate immunity in spontaneous regression/complete resistance mice. Proc Natl Acad Sci USA 2006, 103:7753-7758.

5. Hicks AM, Willingham MC, Du W, Pang CS, Old LJ, Cui Z: Effector mechanisms of the anti-cancer immune responses of macrophages in SR/CR mice. Cancer Immun 2006, 6:11.

6. Hudig D, Ewoldt GR, Woodard SL: Proteases and lymphocyte cytotoxic killing mechanisms. Curr Opin Immunol 1993, 5:90-96.

7. Young JD, Podack ER, Cohn ZA: Properties of a purified pore-forming protein (perforin 1) isolated from $\mathrm{H}$-2-restricted cytotoxic T cell granules. J Exp Med 1986, 164:144-155.

8. Podack ER, Young JD, Cohn ZA: Isolation and biochemical and functional characterization of perforin 1 from cytolytic T-cell granules. Proc Natl Acad Sci USA 1985, 82:8629-8633. 
9. Egorov IK, Egorov OS: Detection of new MHC mutations in mice by skin grafting, tumor transplantation and monoclonal antibodies: a comparison. Genetics 1988, 118:287-298.

10. Dunn GP, Old LJ, Schreiber RD: The immunobiology of cancer immunosurveillance and immunoediting. Immunity 2004, 21:137-148.

11. Kim R, Emi M, Tanabe K, Arihiro K: Tumor-driven evolution of immunosuppressive networks during malignant progression. Cancer Res 2006, 66:5527-5536.

12. Moser B, Willimann K: Chemokines: role in inflammation and immune surveillance. Ann Rheum Dis 2004, 63(Suppl 2):ii84-ii89.

13. Hundhausen C, Misztela D, Berkhout TA, Broadway N, Saftig P, Reiss K, Hartmann D, Fahrenholz F, Postina R, Matthews V, et al.: The disintegrinlike metalloproteinase ADAM10 is involved in constitutive cleavage of CX3CL1 (fractalkine) and regulates CX3CL1-mediated cell-cell adhesion. Blood 2003, 102:1186-1195.

14. Tsou CL, Haskell CA, Charo IF: Tumor necrosis factor-alpha-converting enzyme mediates the inducible cleavage of fractalkine. $J$ Biol Chem 2001, 276:44622-44626.

15. Garton KJ, Gough PJ, Blobel CP, Murphy G, Greaves DR, Dempsey PJ, Raines EW: Tumor necrosis factor-alpha-converting enzyme (ADAM17) mediates the cleavage and shedding of fractalkine (CX3CL1). J Biol Chem 2001, 276:37993-38001.

16. Gale LM, McColl SR: Chemokines: extracellular messengers for all occasions? Bioessays 1999, 21:17-28.

17. Beck C, Schreiber H, Rowley D: Role of TGF-beta in immune-evasion of cancer. Microsc Res Tech 2001, 52:387-395.

18. Rivoltini L, Carrabba M, Huber V, Castelli C, Novellino L, Dalerba P, Mortarini R, Arancia G, Anichini A, Fais S, Parmiani G: Immunity to cancer: attack and escape in Tlymphocyte-tumor cell interaction. Immunol Rev 2002, 188:97-113.

19. Oble DA, Loewe R, Yu P, Mihm MC Jr: Focus on TILs: Prognostic significance of tumor-infiltrating lymphocytes in human melanoma. Cancer Immunity 2009, 9:3.

20. Sato E, Olson SH, Ahn J, Bundy B, Nishikawa H, Qian F, Jung bluth AA, Frosina D, Gnjatic S, Ambrosone C, Kepner J, Odunsi T, Ritter G, Lele S, Chen YT, Ohtani H, Old LJ, Odunsi K: Intraepithelial CD8+ tumorinfiltrating lymphocytes and a high CD8+/regulatory T-cell ratio are associated with favorable prognosis in ovarian cancer. Proc Natl Acad SciUSA 2005, 102:18538-18543.

21. Liakou Cl, Narayanan S, Ng Tang D, Logothetis CJ, Sharma P: Focus on TILs: Prognostic significance of tumor-infiltrating lymphocytes in human bladder cancer. Cancer Immun 2007, 7:10.

22. Dunn GP, Dunn IF, Curry WT: Focus on TILs: Prognostic significance of tumor-infiltrating lymphocytes in human glioma. Cancer Immunity 2007, 7:12.

23. Ohtani H: Focus on TILs: Prognostic significance of tumor-infiltrating lymphocytes in human colorectal cancer. Cancer Immunity 2007, 7:4

24. Galon J, Costes A, Sanchez-Cabo F, Kirilovsky A, Mlecnik B, Lagorce-Page's C, Tosolini M, Camus M, Berger A, Wind P, Zinzindohoue F, Bruneval P. Cugnenc PH, Trajanoski Z, Fridman WH, Page's Fa: Type, Density, and Location of Immune Cells Within Human Colorectal Tumors Predict Clinical Outcome. Science 2006, 313:1960-1964.

\section{Pre-publication history}

The pre-publication history for this paper can be accessed here: http://www.biomedcentral.com/1471-2407/10/179/prepub

\section{doi: $10.1186 / 1471-2407-10-179$}

Cite this article as: Riedlinger et al, The spectrum of resistance in SR/CR mice: the critical role of chemoattraction in the cancer/leukocyte interaction BMC Cancer 2010, 10:179

\section{Submit your next manuscript to BioMed Central} and take full advantage of:

- Convenient online submission

- Thorough peer review

- No space constraints or color figure charges

- Immediate publication on acceptance

- Inclusion in PubMed, CAS, Scopus and Google Scholar

- Research which is freely available for redistribution

Submit your manuscript at www.biomedcentral.com/submit
C Biomed Central 